\title{
Postquench Dynamics of Quantum Dot Proximitized to Superconducting Lead
}

\author{
B. BARAN*, R. TARANKo AND T. DOMAŃski
}

Institute of Physics, M. Curie-Skłodowska University, pl. Marii Curie-Sktodowskiej 1, 20-031 Lublin, Poland

Doi: 10.12693/APhysPolA.138.691

*e-mail: bartlobaran@kft.umcs.lublin.pl

\begin{abstract}
Quantum systems driven from their equilibrium phase can exhibit a rich variety of new phenomena. In this paper, we consider such nonequilibrium dynamics of the single-level quantum dot suddenly embedded between the superconducting and metallic reservoirs. We also investigate three different types of the quantum quenches, examining the dynamics of the charge occupancy of a quantum dot and time-dependent transport through its subgap bound states.
\end{abstract}

topics: superconducting proximity effect, quantum dots, nonequilibrium dynamics

\section{Introduction}

Upon abruptly perturbing superconductors or other objects, such as magnetic impurities, nanowires or atomic islands proximitized to them, one can trigger nontrivial time-dependence of the complex order parameter [1] as has been detected by ultrafast optical measurements in bulk systems [2] and other empirical methods in ultracold superfluids [3]. Of particular interest has recently been the nonequilibrium dynamics originating from quantum quenches, where some initially prepared state $\left|\Psi\left(t_{0}\right)\right\rangle$ of a physical system described by the Hamiltonian $H_{0}$ undergoes evolution to $|\Psi(t)\rangle=\exp (-\mathrm{i} H t / \hbar)\left|\Psi\left(t_{0}\right)\right\rangle$ which, at later times $t>t_{0}$, is described by a different Hamiltonian $H \neq H_{0}$. These phenomena, including the dynamical quantum phase transitions [4], can be conveniently explored in nanoscopic heterostructures owing to a handful of experimental methods allowing to change the model parameters $H_{0} \rightarrow H$ in a controllable manner.

We consider here such nonequilibrium dynamics for a single quantum dot suddenly embedded between a normal metal and an $s$-wave superconductor (Fig. 1). We investigate transient phenomena observable in the charge current flowing through this heterostructure and investigate three different types of quantum quenches, assuming that initially the components of our system are separated from one another. In particular, we analyze a relaxation process and study a response of our setup to a sudden change of its parameters. These possible changes can be driven, e.g., by the gate voltage jump [5-7], optical driving $[8,9]$, phonon-induced processes [10], sudden coupling between the constituents [11] or quantum dynamics of the initially entangled states [12].

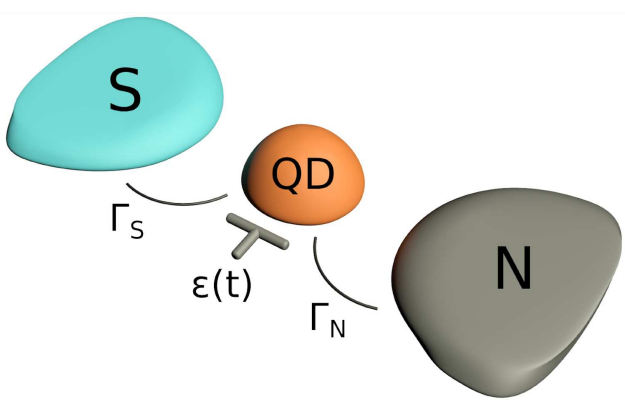

Fig. 1. Setup comprising the quantum dot (QD) coupled to the normal $(\mathrm{N})$ and superconducting $(\mathrm{S})$ electrodes.

This paper is organized as follows. In Sect. 2, we introduce the microscopic model and briefly discuss basic methodological details. Next, in Sect. 3.1, we consider the quench caused by abrupt detuning of the chemical potential in metallic lead $\mu_{\mathrm{N}}$. In Sect. 3.2, we address another kind of quench caused by a rapid jump of the quantum dot energy level and, finally, in Sect. 3.3, we investigate a periodic driving imposed at some time by an external gate potential (of sinusoidal shape) on the quantum dot energy level.

\section{Model and methodology}

Let us consider the uncorrelated quantum dot coupled on one side to the metallic $(\mathrm{N})$ and on the other side to the superconducting (S) leads. This system can be represented by the Hamiltonian

$$
H=\sum_{\beta=\mathrm{N}, \mathrm{S}}\left(H_{\beta}+H_{\beta-\mathrm{QD}}\right)+H_{\mathrm{QD}} .
$$


The normal metallic lead is described by the free fermion gas $H_{\mathrm{N}}=\sum_{k \sigma} \varepsilon_{k \sigma} c_{k \sigma}^{\dagger} c_{k \sigma}$, where the summation is over the wave vector $k$ and spin $\sigma=\{\uparrow, \downarrow\}$. As usually, $d_{\sigma}, c_{k \sigma}\left(d_{\sigma}^{\dagger}, c_{k \sigma}^{\dagger}\right)$ stand for the annihilation (creation) operators describing electrons of the quantum dot and metallic lead, respectively. The quantum dot (QD) can be described by $H_{\mathrm{QD}}=\sum_{\sigma} \varepsilon_{d} d_{\sigma}^{\dagger} d_{\sigma}$, where $\varepsilon_{d}$ is the energy level.

Its hybridization with external leads is given by

$$
H_{\mathrm{N} / \mathrm{S}-\mathrm{QD}}=\sum_{k / q \sigma} t_{k / q}\left(c_{k / q \sigma}^{\dagger} d_{\sigma}+\text { h.c. }\right),
$$

where $t_{k / q}$ denotes a coupling to the metallic/superconducting lead. We consider here the BCS-type superconducting electrode

$$
H_{\mathrm{S}}=\sum_{q \sigma} \varepsilon_{q \sigma} c_{q \sigma}^{\dagger} c_{q \sigma}^{\dagger}-\sum_{q}\left(\Delta_{\mathrm{sc}} c_{q \uparrow}^{\dagger} c_{q \downarrow}+\text { h.c. }\right)
$$

where $\Delta_{\text {sc }}$ denotes the pairing gap.

We restrict our considerations to the wide-band limit, assuming the constant values of both couplings

$$
\Gamma_{\mathrm{N} / \mathrm{S}}=2 \pi \sum_{k / q}\left|t_{k / q}\right|^{2} \delta\left(\varepsilon-\varepsilon_{k / q \sigma}\right) .
$$

For simplicity, we shall treat $\Delta_{\mathrm{sc}}$ as the largest energy scale, focusing on processes which occur in a subgap regime (engaging only the electronic states deep inside the pairing gap). Under such conditions, the fermionic degrees of freedom of the superconducting lead can be integrated out [13], owing to a bilinear structure of the model Hamiltonian (1). Low-energy physics of the proximitized quantum simplifies then to $H_{\mathrm{S}}+H_{\mathrm{S}-\mathrm{QD}}+H_{\mathrm{QD}} \approx H_{\mathrm{eff}}$, where [14]:

$$
H_{\mathrm{eff}} \equiv \sum_{\sigma} \varepsilon_{d} d_{\sigma}^{\dagger} d_{\sigma}-\left(\frac{\Gamma_{\mathrm{S}}}{2} d_{\uparrow}^{\dagger} d_{\downarrow}^{\dagger}+\text { h.c. }\right)
$$

and $\Gamma_{\mathrm{S}} / 2$ can be interpreted as the effective on-dot pairing potential. Systematic corrections around this limiting case have been discussed, e.g., [15].

Our main purpose is to determine the timedependent charge currents $j_{\beta \sigma}(t)$ and occupancy of the quantum dot $n_{d \sigma}(t)$ induced by transient effects and by quantum quenches. The current $j_{\mathrm{N} \sigma}(t)$ between the normal lead and QD can be derived from the Heisenberg equation, and it reads

$$
j_{\mathrm{N} \sigma}=2 \operatorname{Im}\left(\sum_{k} t_{k}\left\langle d_{\sigma}^{\dagger}(t) c_{k \sigma}(t)\right\rangle\right),
$$

where $\langle\ldots\rangle$ denotes the statistical average. For the uncorrelated QD, the explicit formula for timeevolving operators can be expressed by

$$
\begin{gathered}
c_{k \sigma}(t)=c_{k \sigma}(0) \exp \left(-\mathrm{i} \int_{0}^{t} \varepsilon_{k \sigma}\left(t^{\prime}\right) \mathrm{d} t^{\prime}\right) \\
-\mathrm{i} \int_{0}^{t} \mathrm{~d} t^{\prime} t_{k \sigma} d_{\sigma}\left(t^{\prime}\right) \exp \left(-\mathrm{i} \int_{t^{\prime}}^{t} \varepsilon_{k \sigma}\left(t^{\prime \prime}\right) \mathrm{d} t^{\prime \prime}\right) .
\end{gathered}
$$

In particular, in the wide-band limit approximation one obtains [16]:

$$
\begin{aligned}
& j_{\mathrm{N} \sigma}(t)= \\
& 2 \operatorname{Im}\left(\sum_{k} t_{k} \mathrm{e}^{-\mathrm{i} \varepsilon_{k \sigma} t}\left\langle d_{\sigma}^{\dagger}(t) c_{k \sigma}(0)\right\rangle-\frac{\mathrm{i} \Gamma_{\mathrm{N}}^{\sigma}\left\langle n_{d \sigma}(t)\right\rangle}{2}\right)
\end{aligned}
$$

The other current $j_{\mathrm{S} \sigma}(t)$, flowing between QD and the superconducting lead, can be determined using the charge conservation law $\frac{\mathrm{d}}{\mathrm{d} t} n_{d \sigma}=j_{\mathrm{N} \sigma}(t)+j_{\mathrm{S} \sigma}(t)$. Applying the equation of motion approach to the original model (1), we have previously checked [16] that such a constraint is indeed satisfied.

To determine the time-dependent quantities, we have solved numerically the set of coupled equations for $n_{d \sigma}(t),\left\langle d_{\uparrow}^{\dagger}(t) d_{\downarrow}^{\dagger}(t)\right\rangle,\left\langle d_{\uparrow}^{\dagger}(t) c_{k \uparrow}(0)\right\rangle$, $\left\langle d_{\downarrow}^{\dagger}(t) c_{k \downarrow}^{\dagger}(0)\right\rangle, \quad\left\langle d_{\uparrow}^{\dagger}(t) c_{k \downarrow}(0)\right\rangle$ and $\left\langle d_{\downarrow}^{\dagger}(t) c_{k \uparrow}^{\dagger}(0)\right\rangle$, respectively. Their specific expressions have been discussed in [17].

In our calculations, we set $e=\hbar=1$, so that all energies, currents and time are expressed in units of $\Gamma_{\mathrm{S}}, e \Gamma_{\mathrm{S}} / \hbar$ and $\hbar / \Gamma_{\mathrm{S}}$, respectively. We also treat the chemical potential of the superconducting lead as a convenient reference energy $\left(\mu_{\mathrm{S}}=0\right)$ and performed the calculations for zero temperature.

\section{Numerical results}

Now we discuss time-dependence of $n_{d \sigma}(t)$, $j_{\mathrm{N} \sigma}(t)$ and $j_{\mathrm{S} \sigma}(t)$ induced in response to different quench scenarios. We assume that all constituents of the system are disconnected up to $t=0$, i.e., $\quad \Gamma_{\beta}(t<0)=0$. At $t=0^{+}$the quantum dot is abruptly coupled to both external leads and the ensuing relaxation processes manifest themselves through transient phenomena. In Sect. 3.1 we consider the case when the chemical potential of the normal lead is abruptly detuned, safely after the system achieved its stationary state. The case when the QD energy level is suddenly disturbed by an external gate potential is studied in Sect. 3.2 and, finally, in Sect. 3.3 we study the case with the periodically oscillating QD energy level. We compare the results obtained when such oscillations are induced at $t=0^{+}$and after some delay, respectively.

\subsection{Rapid detuning of the chemical potential}

Assuming the initial QD occupancy $n_{d \sigma}(0)=0$, we discuss transient oscillations of $n_{d \sigma}(t), j_{\mathrm{N} \sigma}(t)$ and $j_{\mathrm{S} \sigma}(t)$ caused by the abrupt couplings of QD to the external leads (Fig. 2). We notice that these oscillations are dumped due to the coupling to a continuous spectrum of the normal lead $[17,18]$, thus

$$
\begin{aligned}
& n_{d \sigma}(t)=\sin ^{2}\left(\Gamma_{\mathrm{S}} t / 2\right) \exp \left(-\Gamma_{\mathrm{N}} t\right) \\
& j_{\mathrm{S} \sigma}(t)=\frac{1}{2} \Gamma_{\mathrm{S}} \sin \left(\Gamma_{\mathrm{S}} t\right) \exp \left(-\Gamma_{\mathrm{N}} t\right), \\
& j_{\mathrm{N} \sigma}(t)=\frac{1}{2} \Gamma_{\mathrm{N}} \sin \left(\Gamma_{\mathrm{S}} t\right) \exp \left(-\Gamma_{\mathrm{N}} t\right) .
\end{aligned}
$$



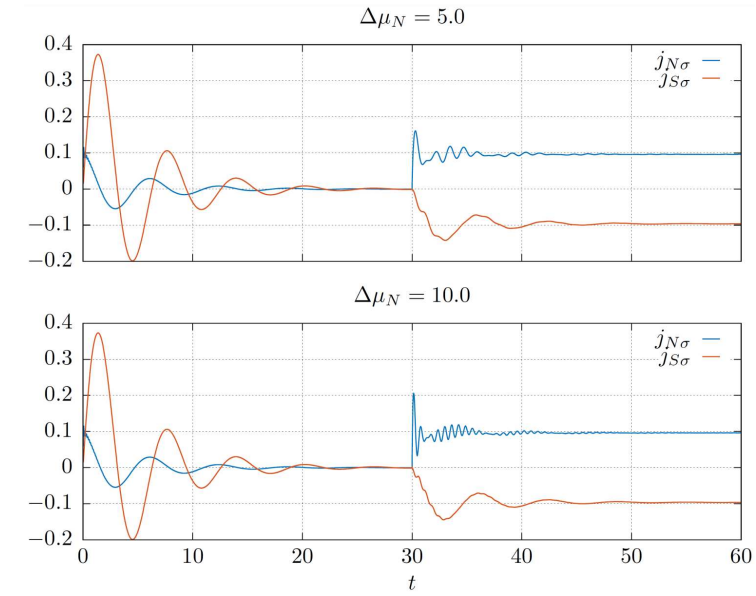

Fig. 2. Time-dependent currents in the system with the jump of a chemical potential level $\mu_{\mathrm{N}} \rightarrow$ $\mu_{\mathrm{N}}+\Delta \mu_{\mathrm{N}}$ at $t \geq 30$, obtained for the parameters: $\mu_{\mathrm{N}}=0, \Gamma_{\mathrm{N}}=0.2, \varepsilon_{d}=0$.

After a sudden change of the chemical potential, the time-dependent current looks quite different. It is known [19-21] that the current flowing through the QD coupled between both normal leads responds to such an abrupt change of the bias voltage by coherent oscillations with the frequency $\omega=\left|\mu-\varepsilon_{d}\right|$.

In our case, the proximitized QD is characterized by a pair of in-gap bound states formed at $\pm \Gamma_{\mathrm{S}} / 2$, therefore we observe a superposition of two currents contributing to $j_{\mathrm{N} \sigma}(t)$ with the frequencies $\omega_{1,2}=\mu \pm \Gamma_{\mathrm{S}} / 2$, respectively. As a result, the oscillating current has the high frequency component $\omega=\mu$ superimposed with the lower frequency beats (with the time period $T=2 \pi$ ). Contrary to this behavior, we notice that the current $j_{\mathrm{S} \sigma}$ flowing between the superconducting lead and QD oscillates with only the lower frequency. A difference between these currents leads to the time-dependent occupancy, obeying the local charge conservation $\frac{\mathrm{d}}{\mathrm{d} t} n_{d \sigma}=j_{\mathrm{N} \sigma}(t)+j_{\mathrm{S} \sigma}(t)$.

\subsection{Rise of QD's energy level}

Let us now investigate the case of an abrupt change of the energy level $\varepsilon_{d} \rightarrow \varepsilon_{d}+\Delta \varepsilon_{d}$ at some time $\left(t=30 / \Gamma_{\mathrm{S}}\right)$, safely distant after the transient processes. Until that moment, all observables reveal an oscillatory behavior (see the formulae in Sect. 3.1) approaching exponentially their stationary values. After a rapid jump of $\varepsilon_{d}$ from zero value to $\Delta \varepsilon_{d}$, we observe the emergence of a completely different time evolution for all observables (Fig. 3). In particular, the period of oscillations is now controlled by $\varepsilon_{d}$ and it equals $2 \pi / \sqrt{\Gamma_{\mathrm{S}}^{2}+4\left(\Delta \varepsilon_{d}\right)^{2}}$ [17].

After a sufficiently long time interval, the oscillations gradually decay and the currents $j_{\mathrm{S} \sigma}, j_{\mathrm{N} \sigma}$ tend towards their stationary vanishing values (because $\mu_{\mathrm{N}}=\mu_{\mathrm{S}}$ ). We note, however, that the damping factor describing $j_{\mathrm{S} \sigma}(t)$ is quite different for

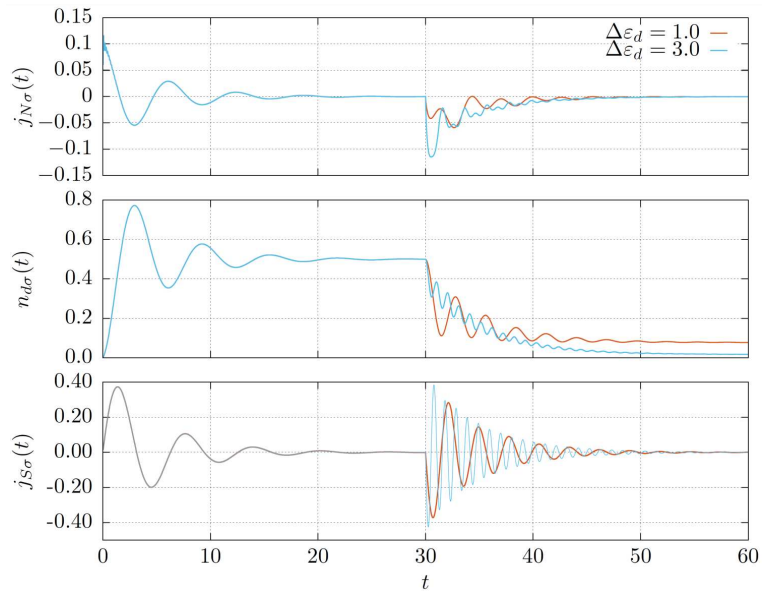

Fig. 3. Time-dependent currents and QD occupation in the system with the jump of energy level $\varepsilon_{d \sigma} \rightarrow \varepsilon_{d \sigma}+\Delta \varepsilon_{d}$ of $\mathrm{QD}$ at $t \geq 30$, obtained for the parameters: $\mu_{\mathrm{N}}=0, \Gamma_{\mathrm{N}}=0.2, \varepsilon_{d \sigma}=0$.

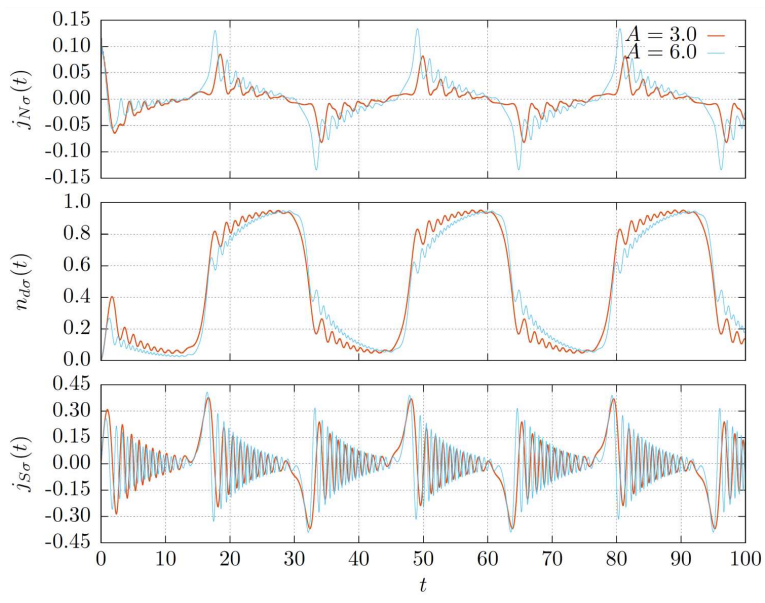

Fig. 4. Time-dependent currents and QD occupation in the case when $\varepsilon_{d \sigma}(t)$ and coupling between all parts of the system were turned on in $t=0^{+}$, obtained for $\omega=0.2, \mu_{\mathrm{N}}=0, \Gamma_{\mathrm{N}}=0.2$.

the transients appearing just after the abrupt coupling of all parts of the system and the ones appearing after the jump of $\varepsilon_{d}$. The first is $\frac{\Gamma_{\mathrm{S}}^{2}}{2} \exp \left(-\Gamma_{\mathrm{N}} t\right) \sqrt{\Gamma_{\mathrm{S}}^{2}+4 \varepsilon_{d}^{2}}$ and depends on $\varepsilon_{d}$ [17] but the second is independent of $\Delta \varepsilon_{d}$ and equals $\sim \exp \left(-\Gamma_{\mathrm{N}} \bar{t}\right) / 2.24$ (here $\bar{t}$ denotes the time measured from the $\varepsilon_{d}$ jump moment). Similarly, also the time-dependent occupancy of QD evolves to the stationary values dependent on $\Delta \varepsilon_{d}$.

\subsection{Periodic driving}

Finally, let us consider the case of the oscillating QD's energy level, $\varepsilon_{d}(t)=A \sin (\omega t)$. In Fig. 4 , we present $j_{\mathrm{N} \sigma}(t), n_{d \sigma}(t)$ and $j_{\mathrm{S} \sigma}(t)$ obtained numerically for the case when the periodic driving is combined with the transient effects switched at $t=0^{+}$. For comparison, in Fig. 5, we consider the influence of oscillations $\varepsilon_{d}$ switched 


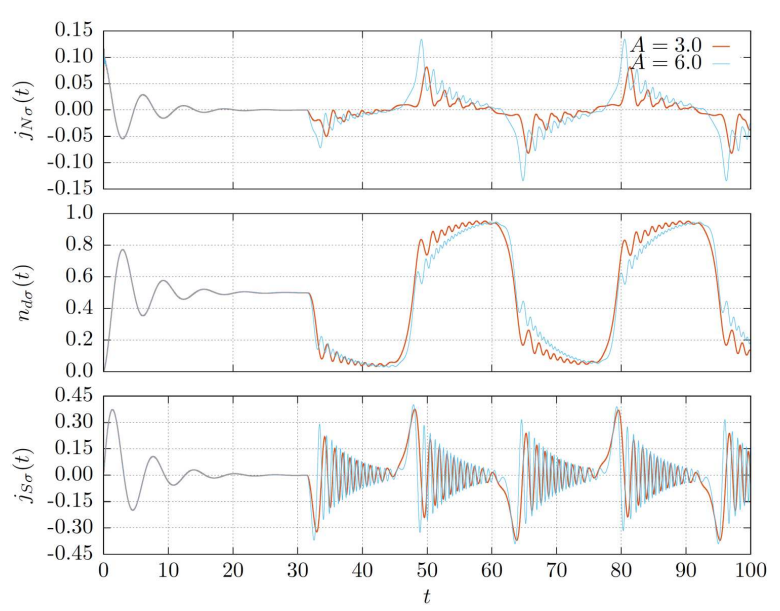

Fig. 5. Time-dependent currents and QD occupation in the system with the external perturbation of QD's energy level with the amplitude $A$ and the frequency $\omega=0.2$ turned on $t=12 \pi$, obtained for $\mu_{\mathrm{N}}=0, \Gamma_{\mathrm{N}}=0.2$.

after the system has nearly finished the relaxation effects. In general, we notice that time evolution of the considered observables is nearly identical for both cases. For this reason, the periodic driving could hence be well described by the Floquet-type analysis [22], using physical observables averaged over the period.

\section{Summary}

We have investigated the transient phenomena superimposed with the dynamical effects induced by three representative types of the quantum quenches observable in the quantum dot hybridized with the metallic and superconducting leads. We have shown that these time-dependent quantities are characterized by the dumped quantum oscillations with the period sensitive to the in-gap quasiparticle energies and their distance to the chemical potential. These phenomena should be observable experimentally and might give insight into the dynamics of quantum systems far away from their equilibrium situations. Our considerations could be further generalized to heterostructures with more numerous electrodes and/or different arrangements of the quantum dots.

\section{Acknowledgments}

This work has been supported by the National Science Centre (NCN, Poland) under the grant number UMO-2017/27/B/ST3/01911.

\section{References}

[1] T. Cui, M. Schütt, P. Orth, R. Fernandes, Phys. Rev. B 100, 144513 (2019).

[2] R. Shimano, N. Tsuji, Ann. Rev. Condens. Matter Phys. 11, 103 (2020).

[3] T. Langen, R. Geiger, J. Schmiedmayer, Ann. Rev. Condens. Matter Phys. 6, 201 (2015).

[4] M. Heyl, Rep. Prog. Phys. 81, 054001 (2018).

[5] A.E. Antipov, Q. Dong, E. Gull, Phys. Rev. Lett. 116, 036801 (2016).

[6] I. Krivenko, J. Kleinhenz, G. Cohen, E. Gull, Phys. Rev. B 100, 201104 (2019).

[7] D.M. Kennes, V. Meden, Phys. Rev. B 85 , 245101 (2012).

[8] K. Oreszczuk, M. Goryca, W. Pacuski, T. Smolenski, M. Nawrocki, P. Kossacki, Phys. Rev. B 96, 205436 (2017).

[9] R. Vasseur, J.E. Moore, Phys. Rev. Lett. 112, 146804 (2014).

[10] A. Nysteen, P. Kaer, J. Mork, Phys. Rev. Lett. 110, 087401 (2013).

[11] K. Wrzesniewski, I. Weymann, Phys. Rev. $B$ 100, 035404 (2019).

[12] N.S. Maslova, P.I. Arseyev, V.N. Mantsevich, Phys. Rev. A 96, 042301 (2017).

[13] J. Bauer, A. Oguri, A.C. Hewson, J. Phys. Condens. Matter 19, 486211 (2007).

[14] J. Baranski, T. Domanski, J. Phys. Condens. Matter 25, 435305 (2013).

[15] T. Meng, S. Florens, P. Simon, Phys. Rev. B 79, 224521 (2009).

[16] A.-P. Jauho, N.S. Wingreen, Y. Meir, Phys. Rev. B 50, 5528 (1994).

[17] R. Taranko, T. Domanski, Phys. Rev. B 98, 075420 (2018).

[18] R. Taranko, T. Kwapinski, T. Domanski, Phys. Rev. B 99, 165419 (2019).

[19] F.M. Souza, Phys. Rev. B 76, 205315 (2007).

[20] E. Perfetto, G. Stefanucci, M. Cini, Phys. Rev. B 78, 155301 (2008).

[21] E. Taranko, M. Wiertel, R. Taranko, J. Appl. Phys. 111, 023711 (2012).

[22] B. Baran, T. Domanski, Phys. Rev. B 100, 085414 (2019). 